\title{
Producción de conocimiento y toma de decisiones. Relaciones entre academia y política pública para las familias en Colombia
}

\author{
José Raúl Ruiz \\ Doctor en Ciencias Sociales Niñez y Juventud. Licenciado en Biología \\ Fundación Universitaria Monserrate. Bogotá, Colombia \\ https:/ / orcid.org/0000-0003-2089-565X・jraulruiz@unimonserrate.edu.co

\section{Sandro Leonardo Munévar-Vargas} \\ Doctor en Comunicación. Licenciado en Filosofía e Historia \\ Fundación Universitaria Monserrate. Bogotá, Colombia \\ https:// orcid.org/0000-0003-3835-6742・ smunevar@unimonserrate.edu.co
}

\section{Resumen}

El artículo presenta los resultados de la investigación en torno a las relaciones entre academia y política pública en familia. Específicamente se trabajó con cuatro universidades del país, que desarrollan programas posgraduales en familia y que a su vez han aportado a las políticas públicas de familia, tanto a nivel local como nacional. Los objetivos del estudio se concretaron en comprender los múltiples vínculos, agentes y contextos en ambos campos, el de la investigación en el campo de familia y su conexión con las decisiones que se toman para el desarrollo de políticas públicas de familia. La metodología se decidió a partir de la tradición hermenéutica y se optó por grupos focales, entrevistas y amplias revisiones de documentos.

Los resultados conllevan a plantear los múltiples perfiles de maestras y maestros quienes investigan con familias, sus aportes y conexiones; asimismo se establece cómo la investigación aporta conocimiento al hacedor de política pública, siendo un elemento crucial y que respalda la toma de decisiones. Igualmente, se asumen aspectos compartidos tanto para quienes investigan, como para quienes diseñan y concretan políticas públicas, aspecto que suma para una de las conclusiones: la dinámica compartida entre academia y política pública.

Palabras clave: Política pública de familia; Estudios de familia; Producción de conocimiento; Academia.

Recibido: 15/01/2021 | Aprobado: 22/04/2021 |Publicado: 01/07/2021

cc) (i) (-) Esta obra está bajo una Licencia Creative Commons Atribución-NoComercial-CompartirIgual 4.0 Internacional.

Financiación o proveniencia del artículo: Artículo derivado de la investigación Relación de los programas posgraduales de familia y la política pública de familia a través de los planes de desarrollo en el período comprendido entre 2001 a 2017 en Colombia, financiada por la Fundación Unimonserrate a través de la convocatoria institucional del segundo semestre de 2017. Investigación con código POS 2017-2 que culminó en el segundo semestre de 2018.

¿Cómo citar este artículo? / How to quote this article?

Ruiz, J. R., y Munévar-Vargas, S. L. (2021). Producción de conocimiento y toma de decisiones. Relaciones entre academia y política pública para las familias en Colombia. Prospectiva. Revista de Trabajo Social e intervención social, (32), 239-258. doi: 10.25100/prts.v0i32.10944. 
Ruiz y Munévar-Vargas

\title{
Knowledge Production and Decision Making. Relationships Between Academy and Public Policy for Families in Colombia
}

\begin{abstract}
The article presents the results of research on the relations between academia and public policy in the family. Specifically, it worked with four universities in the country, which offer postgraduate family programs and which in turn have contributed to public family policies, both locally and nationally. The objectives of the study were concerned with understanding the multiple links, agents and contexts in both fields: of research in the field of family and its connection with the decisions made for the development of public policies concerning family. The methodology was decided from the hermeneutical tradition and opted for focus groups, interviews and extensive document reviews.

The results lead to raise the multiple profiles of teachers who investigate with families, their contributions and connections; it also establishes how research provides knowledge to the public policy maker, being a crucial element that supports decision-making. Likewise, shared aspects are assumed for those who investigate, as well as for those who design and implement public policies, an aspect that adds up to one of the conclusions: the shared dynamics between academia and public policy.

Keywords: Family Public policy; Family studies; Knowledge production; Academia.

Sumario: 1. Introducción, 2. Posicionamiento teórico, 2.1 Los paradigmas determinantes de la política pública, 2.2 La producción y comunicación de conocimiento en el escenario de la política pública de familia, 2.3 Formas de concebir y pensar la familia en la política pública nacional de familia, 3. Metodología, 4. Hallazgos, 4.1 La Universidad produce, articula y resignifica el conocimiento, 4.2 La toma de decisiones para la política pública en familia, 5. Conclusiones, 6. Agradecimientos, 7. Referencias bibliográficas.
\end{abstract}




\section{Introducción}

Varias instituciones del estado colombiano solicitan de forma permanente conceptos a los centros de investigación y comunidades académicas en torno a temas de gran discusión nacional, o que generan controversia e impacto en la vida pública del país. Conceptos sostenidos en la producción de conocimiento que desarrollan universidades y centros especializados de investigación. Dichas solicitudes ayudan en la toma de decisiones para emitir amplios fallos jurídicos o emitir políticas públicas. Es decir, hay vínculos que deben ser develados entre la Academia y el escenario de la toma de decisiones en política pública, muchos nexos que se sustentan en torno a las demandas desarrolladas por la sociedad, requerimientos que exigen soluciones y que de algún modo quedan en promesas. Ya De Sousa-Santos (2000) denuncia cómo el momento histórico que vivimos, con un amplio desarrollo del conocimiento, no es proporcional a las soluciones que exigimos lo seres humanos.

La política pública como ámbito de decisión colectiva supone un mundo de complejidades que abarcan el orden social, político, económico, cultural y retan a la academia ${ }^{21}$ como lectora crítica de las realidades sociales y políticas de las naciones y por ende, demanda a la academia, y a los investigadores para que cuestionen todas aquellas certezas instauradas en una sociedad, y planteen posturas para la dignidad humana.

El artículo presenta los resultados obtenidos del estudio que relaciona la academia productora de conocimiento- con la política pública de familia, que asume decisiones que impactan a amplias comunidades. Resultados que revelan un diálogo abierto y profundo sobre la experiencia de algunos actores del mundo académico con múltiples agentes como: estudiantes de diversos programas de las ciencias sociales y humanas, comunidades de algunas capitales del país, diversas familias en sus territorios y varios agentes que intervienen en el ámbito de la administración pública quienes formulan, implementan y evalúan la política pública de familia en Colombia. Varias de las inquietudes del estudio nacen en el Grupo de Investigación, Estudio de Contextos y Realidades Sociales de la Fundación Unimonserrate en diálogo con la sublínea de investigación, Familia, Educación y Estudios Interdisciplinarios de la Maestría en Familia Educación y Desarrollo y la línea de investigación, Familia, Desarrollo humano y social.

1 Para el presente estudio se asume la academia como las Instituciones de Educación Superior IES, las cuales tienen dentro de sus funciones legales: la investigación (producción de conocimiento y comunicación del mismo), la docencia y la extensión. Es así como para el interés de la investigación se trabajó con cuatros Universidades que presentan programas posgraduales en familia y, que a su vez, son reconocidas por los aportes en el campo de familia. Por ello, la fase de campo se desarrolló con la Universidad de Caldas, la Universidad del Valle, la Universidad de Antioquía y la Fundación Unimonserrate. 


\section{Posicionamiento teórico}

\subsection{Los paradigmas determinantes de la política pública}

Las políticas públicas surgen como campo de estudio de la posguerra, con el propósito de aplicar el método científico a la toma de decisiones gubernamentales. Estas son vistas como un instrumento de gobierno que contribuye a la construcción de la democracia. Ahora bien, a partir del surgimiento de la nueva gestión pública, las políticas públicas se constituyen como una herramienta de gestión para lograr eficiencia económica y efectividad en la solución de problemas considerados como públicos.

Este campo de estudio fue desarrollado en la década de los años 50 por Laswell (1971) y coincide con un periodo que demandaba el uso de una tecnología de gobierno para enfrentar la crisis de la posguerra en los Estados Unidos. Para Laswell, el campo de la política gira en torno a tres ejes principales: la interdisciplinariedad, la aplicación del conocimiento científico y la contribución al fortalecimiento de la democracia.

Posteriormente surgen tres enfoques en el campo de las políticas públicas: el enfoque de toma de decisiones, los estudios sobre la implementación y los estudios de evaluación. A partir de allí se toma a la política pública como un proceso que agrupa tres momentos: la formulación, la implementación y la evaluación. Esta concepción limitada de las políticas fue debatida posteriormente y puso en consideración la simultaneidad de los procesos y la reformulación de las políticas a partir del aprendizaje. Teniendo en cuenta las limitaciones del enfoque cíclico, se plantearon nuevas formas de análisis de la política. Estos enfoques fueron agrupados por Roth-Deubel (2002) en teorías centradas en el Estado, la sociedad y las teorías mixtas.

Una aproximación a la definición de la política pública se puede alcanzar con una visión que muestra el lugar de la ciudadanía en la conformación de los escenarios de toma de decisiones políticas; sin embargo, más que una definición se propone como un lugar de comprensión. Según Lozano (2008):

La perspectiva de la gobernanza nos muestra un escenario de apertura, que reconoce la complejidad y un mayor pluralismo en la elaboración y la implementación de unas políticas públicas que, actualmente, se tienen que elaborar a través de una negociación constante entre diferentes organismos, diferentes niveles de gobierno y diferentes actores privados y o comunitarios. (p. 4)

Desde una representación de la negociación pública:

La política pública hay que comprenderla como el resultado de una acción colectiva que se desarrolla en lo público y de una serie de transacciones políticas, en donde el gobierno 
ya no tiene como único objetivo ejecutar lo planeado, sino también garantizar la coordinación y la cooperación de los actores clave. Lo anterior recalca dos elementos fundamentales del concepto: lo político y lo público. (Torres-Melo y Santander, 2013, p.

56)

En consecuencia, para la investigación lo público es un asunto al que se le da forma de objeto en las políticas públicas, pues genera intereses para los sectores de la comunidad. Se tiende a creer que este objeto está ya formado y que la política pública entra simplemente a organizarlo en términos de decisión frente al objeto; sin embargo, la comprensión del objeto como una construcción social es esencial dentro de los Estados democráticos, y por ello es necesario agendar la discusión en orden a alcanzar una definición del objeto mismo.

Esta relación entre lo político y lo público, la presenta Subirats, Knoepfel, Larrue y Varone (2008) cuando destaca que el contenido y las características institucionales de una acción pública son el resultado de las interacciones entre las autoridades políticoadministrativas y los grupos sociales que causan y/o padecen los efectos negativos de un determinado problema colectivo. Problema que, precisamente, trata de resolver la actuación de los poderes públicos. El «juego» de tales actores depende no sólo de sus intereses respectivos, sino también recursos que logren movilizar para defender sus posiciones con relación a los objetivos, a los instrumentos y a los procesos de elaboración de una intervención pública. Así, dicha dinámica o juego puede darse tanto en la definición del contenido de la política pública, como en la forma en la que ésta se lleva a cabo o se implementa.

Es así como la política pública se estructura a partir de la lucha de poderes e intereses económicos, sociales, políticos e ideológicos de diversos actores en diferentes momentos. De tal manera que el entendimiento de la política pública como un ciclo permite apreciar su desarrollo como proceso y con base en una serie momentos constitutivos, a saber: 1) posicionamiento de un problema en la agenda pública, 2) formulación de acciones dirigidas a mitigar el problema mediante políticas, 3) cristalización de una decisión concreta frente al mismo, 4) implementación de las políticas, y 5) evaluación de éstas. Esta forma de entender las políticas públicas contribuye a entender los momentos bajo los cuales pasa la acción pública, y sirve como modelo pedagógico para su entendimiento. Sin embargo, pese a su matriz racional, se evidencia una serie de falencias para analizar la misma debido a que puede quedar en la simple formulación de propuestas de acción frente a los problemas públicos, a su estricta linealidad que no obedece a los procesos decisorios empíricos y produce incongruencias para entender las incertidumbres en el proceso de política, siendo incapaz de abordar las diferentes implicaciones de los actores en la composición de la política pública. 
Ruiz y Munévar-Vargas

La política pública debe ser considerada también como paradigma, lo que implica el conocimiento de una sociedad a partir de la forma en que asume determinada realidad, en este sentido se puede estudiar con Foucault el devenir de la forma del poder estatal en las sociedades a partir del estudio de la forma en que el Estado intervino en diferentes realidades a través de la historia.

En este análisis que establece la relación entre política pública y academia, se considera pertinente utilizar dos modelos o formas de aproximación a las políticas públicas; la primera ya presentada y que proviene de la tradición de la ciencia racional moderna que brinda elementos importantes para la comprensión; y la segunda, entendiendo la política como paradigma, decisión que se alcanza en el desarrollo mismo del diálogo sostenido durante la investigación con el mundo académico, con las unidades académicas de las universidades participantes del estudio. Como resultado, se considera que las dos formas de análisis son complementarías y que manifiestan la complejidad misma del objeto de estudio.

De esta manera toma sentido la afirmación que, desde diversos actores, entre ellos la comunidad académica, se hace frente a la ausencia de voluntad política para la implementación de determinados discursos de la política pública. En tanto la acción pública alrededor de la familia, tiene como sustento una serie de intereses históricamente construidos que buscan generar exclusiones sistemáticas y que tienen por objetivo construir unarealidad social y elaborar los componentes de lectura de esta. Por lo tanto, hay que destacar la política pública como paradigma que tiene alto contenido explicativo, en tanto brinda herramientas para el análisis de los objetivos y pretensiones que tiene la política pública para construir realidad.

\subsection{La producción y comunicación de conocimiento en el escenario de la política pública de familia}

El gobierno colombiano formuló la política pública de familia para los años 2012-2022, en cabeza del Ministerio de Salud y Protección Social, por medio de la conformación de la Mesa nacional de familias, integrada por entidades públicas, organizaciones sociales y universidades con trayectoria reconocida en el campo de familia. En este sentido, se realizaron charlas magistrales, discusiones, revisiones documentales y normativas. Todo esto valiéndose de la metodología de marco lógico avalada por el Departamento Nacional de Planeación.

Para cumplir con el propósito de la conformación de la mesa nacional, se realizaron talleres en el orden departamental, municipal y distrital, y se contó con la participación de servidores públicos, contratistas, sociedad civil organizada, universidades públicas y privadas y la comunidad religiosa en general. 
Ruiz y Munévar-Vargas

Es necesario destacar el rol activo y articulador de varias universidades participantes en el proceso de formulación de la política pública nacional de familia 2012-2022, en el sentido de aportar conocimientos técnicos y conceptuales para la formulación del problema principal, identificación de las causas, consecuencias, objetivos y fines, necesarios para articular el conocimiento académico con la solución de problemas socialmente relevantes, en este caso, los relacionados con la familia colombiana.

En este escenario, las Instituciones de Educación Superior IES, como productoras de conocimiento, a partir de la investigación, convocan a considerar dentro de sus lecturas de realidad las interrelaciones entre lo local y lo global. Sumado a ello, la globalización y su impronta en términos de comunicación, ha llevado al desarrollo de amplias redes de información. Esos modos de comunicación han comprimido el espacio y el tiempo, fenómeno que no es ajeno a los procesos de producción de conocimiento (Touraine, 2006). Es decir, las formas de comunicar el conocimiento, las cuales pasan por consensos e intereses (especialmente económicos, pero también políticos) de orden mundial, nacional e institucional. Es así como los diversos productos derivados de la investigación se insertan en una lógica en la cual la rapidez y vigencia -enmarcada en la inmediatez- implica colocar lo producido por parte de los investigadores, en una temporalidad, en la provisionalidad y que refiere un mercado para comunicar, mercado que ha definido lugares, lenguajes y escenarios más taquilleros (revistas reconocidas, grupos, idiomas para publicar, entre otros). Todo ello no es ajeno en el campo de familia y sus investigaciones.

Anexo a ello, la tensión permanente entre la homogenización -incluso la de los conglomerados de sujetos- y la exacerbación de variadas individualidades que se enmarcan en posturas ideológicas, religiosas, étnicas, de múltiples diversidades, configura una postura antagónica.

Frente a lo explicitado, la academia debe asumir los nuevos retos de comprender los hechos sociales, bajos diferentes referentes, que deben desmarcarse de modos únicos de comprender a los sujetos y establecer la significación de las experiencias de vida y las formas de organización que los sujetos deciden asumir. Formas de organización que, para el caso de la familia contemporánea, fracturan relatos como los del binarismo de género, la asociación familiar monógama, el descentramiento de la mujer como centro y eje de la familia, o el establecimiento de vínculos perpetuos. Es decir, partiendo de la realidad como un todo orgánico en movimiento, la configuración de las formas de vivir y el sentido que le damos, es cambiante. Metamórfico, camaleónico, en el tiempo y en el espacio.

De tal manera que el papel de la comunidad académica (y de las y los investigadores) en la producción de conocimiento y el acercamiento a diversas realidades, está atravesado por los supuestos ideológicos institucionales y por la subjetividad de sus investigadoras e investigadores. Subjetividad que implica: prejuicios, formas de pensar y sentir, que 
estipulan el ejercicio mismo de investigar, y el cual transita por idealizaciones temporales de teorías, movimientos y autores junto con determinantes estructurales como: la politización misma de la ciencia, la hegemonía de pretensiones objetivas, que a su vez se establecen por modelos de racionalismo instrumental que híper valoran formas de pensamiento cuantitativo. Basta con ver los respaldos de Minciencias a un tipo de ciencias y de producción científica, frente a la forma como se desestima la ciencia social y humana, menosprecio que conlleva a un menor valor de lo producido en estas últimas ciencias, con las consecuencias que debemos cargar quienes investigamos en dichos campos científicos. Sumado a lo anterior, la impronta de la institucionalidad es otra carga para la investigación y quienes ejercen dicha tarea, varias IES y centros de investigación asumen posturas en el modo de concebir la familia, los sujetos, las comunidades, y ello se debe reflejar en las investigaciones que apoyan. Es decir, se investiga bajo intereses de diverso orden.

El modo como circula el conocimiento, la red compleja de movimiento de este, así como los momentos y sujetos participantes en los procesos de producción, es atravesado por aspectos de diverso orden, que para el caso de la relación ciencia-política asume un tipo de sociedad (Delatin, Texeira y Sandroni, 2015). Sociedad que para el caso del tema a tratar toma un tipo de familia, un modo común de organización y una sedimentación de pensar y reconocer la Familia. No obstante, las universidades y los centros de investigación revelan aspectos distantes de la noción única de familia. Dichos develamientos se presentan en los amplios ejercicios de producción de conocimiento, los cuales se estructuran en la organización interna de líneas de investigación, grupos, semilleros y realización de tesis, como productos de trabajos de grado de los programas que se inscriben en pensar la familia.

\subsection{Formas de concebir y pensar la familia en la política pública nacional de Familia}

La política pública en familia comienza por situar a la familia a partir del marco de los derechos humanos y bajo referentes internacionales. Con ello desarrolla la base para reconceptualizar la familia procurando articular lo legal con la dimensión social de la familia como sujeto colectivo. Si bien la constitución política colombiana reconoce que la familia es una organización social fundamental para la supervivencia del individuo en todas las etapas de su vida, y su incidencia en la sociedad colombiana, también se establece de forma reveladora la singularidad de las familias colombianas en la actual política nacional de familias. Propiedad que está marcada por la diversidad de todo orden: cultural, social, económica y política. De esta forma, aparecen agentes externos que se vinculan con las familias colombianas.

Por otro lado, en la política pública de familia se anuncian y reconocen agentes internos en el ámbito de la familia: las infancias, las juventudes, las mujeres, las personas mayores. Igualmente, se consideran condiciones tales como la discapacidad, la diversidad 
Ruiz y Munévar-Vargas

étnica y cultural. No obstante, es importante resaltar cómo la amplitud de la política de familia y con varios aspectos innovadores, ocasiona otras cuestiones que merecen ser ampliadas.

Cuestiones para resaltar como la proyección del cuidado, como política. La mirada a múltiples tipos de familias y a fenómenos como la migración, el desplazamiento y el desarraigo. En este sentido, la Corte Constitucional de Colombia se pronunció mediante las Sentencias T-070/15 (C.C., T-070/15, 2015) y T-292/16 (C.C., T-292/16, 2016) según las cuales se ratificó la protección constitucional a los diferentes tipos de familia y se prohibió la discriminación según su naturaleza, así como la protección a los diferentes vínculos familiares. Los fallos proferidos en estas sentencias están soportados en los derechos de igualdad, así como la protección de los diferentes tipos de familia. En este sentido La Corte ha dicho que se entiende por familia,

aquella comunidad de personas emparentadas entre sí por vínculos naturales o jurídicos, que funda su existencia en el amor, el respeto y la solidaridad, y que se caracteriza por la unidad de vida o de destino que liga íntimamente a sus integrantes más próximos. (C.C., T-070/15, 2015, p. 1)

En este escenario, y a partir de una serie de acciones colectivas de la llamada comunidad LGBTI que datan del año 2010, aproximadamente, se configura todo un camino de recursos legales que finalizan con la ponencia favorable a la adopción de niños por parte de parejas del mismo sexo. Adicionalmente, la Corte Constitucional amplía la noción de familia, respecto al vínculo único considerado en la constitución nacional el cual se establece entre un hombre y una mujer. Dicha ampliación se extiende para parejas del mismo sexo, y se desarrolla a partir de la Sentencia C-577/11 (Espinosa, Rivera y Ruiz, 2016).

Posteriormente, para el mes de enero del año 2015 la Corte Constitucional a través del Magistrado Jorge Iván Palacio comenzó una discusión, la cual suscitó múltiples disputas en torno a la forma de concebir y comprender la familia. Dicha cuestión, se complejizó cuando se recibieron los conceptos -que habían sido solicitados previamente por la corte- de varias instituciones de orden gubernamental, ONG e instituciones de educación superior IES, a las cuales se les instó a emitir un concepto en torno a la adopción de niños y niñas por parte de parejas del mismo sexo y su conveniencia, o no para los infantes. Los conceptos emitidos permitirían la toma de decisión a presentar por parte del magistrado ponente Iván Palacio (Jiménez, 2015). Cabe anotar que los conceptos expresados por las universidades, para el momento, recogían posturas en torno a temáticas del sexo, el género, la familia, pero con énfasis en los derechos de niños, niñas y padres de la llamada comunidad de gais y lesbianas. 
Los argumentos esbozados, a favor y en contra se sostenían en evidencia empírica y en la discusión de grandes marcos teóricos emanados de diversas posturas de las ciencias sociales y humanas. Marcos teóricos cruzados por formas de concebir y pensar la familia, por parte de investigadores y por supuesto afiliados a centros de investigación. Desde allí ensalzaban en su momento posturas tradicionales y emergentes de concebir la familia, y el vínculo que tienen los sujetos en dicho entramado social. Las posturas se volvieron más antagónicas cuando a partir de concepciones de algunas universidades se defiende la iniciativa de un modo de familia, sostenida en el artículo 42 de la Constitución nacional donde se expresa explícitamente la conformación de la familia en Colombia a partir del vínculo entre "un hombre y una mujer" llevando a proponer para el año 2016 la convocatoria a un referendo que pretendía aprobar la adopción de niños y niñas solo y exclusivamente por parte de parejas heterosexuales (Guevara y Ortiz, 2016), de parte de la entonces senadora Vivian Morales. La senadora en mención pretendía añadir al artículo 44 de la Constitución -que refiere los derechos fundamentales de los niños- una medida de protección la cual se sostenía en garantizar la configuración de la familia heterosexual, como único modo de asociación familiar. Acciones como están negaban la posibilidad de la diversidad familiar, de la diversidad humana y su autónoma forma de generar familias.

\section{Metodología}

El estudio procuró reconstruir las experiencias significativas de diversos actores en la configuración de la política pública de familia en diferentes ciudades del país, tomando como escenario las unidades académicas universitarias con programas de posgrado en el campo de Familia ubicadas en Manizales, Cali, Bogotá y Medellín. En estas cuatro ciudades, se realizó un diálogo con académicos (maestros y maestras) quienes han participado en investigaciones en estudios de familia y en construcción de política pública, así como desplegadas relaciones con diferentes actores académicos e investigativos, comunidades y organizaciones localesy nacionales. Las universidades con las cuales se logró la conexión fueron especialmente de unidades académicas de Trabajo Social y de programas que ofrecían formaciones posgraduales en familia. De allí se contactaron equipos de maestras y maestros que hacían parte de dichos programas de posgrado. Es así como se logró obtener el desarrollo de entrevistas con maestras y maestros que hacían parte de los programas mencionados. Del mismo modo se logró desarrollar grupos focales, uno en cada Universidad, con el acompañamiento de maestras y maestros que desarrollan su labor en el campo de familia. La formación de los participantes era de maestría y varios doctores y doctoras, todos con experiencia en la investigación con familia y consultores en el mismo campo. Sumado a lo anterior, se logra la participación de una candidata a la vicepresidencia de la República, quien además había sido integrante del Senado y hacedora de política pública con vasta experiencia en el campo de familia. 
Ruiz y Munévar-Vargas

Por lo anterior se acudió a la tradición hermenéutica. De acuerdo con Gadamer (1993), la hermenéutica reconoce la importancia de la experiencia del sujeto, ya que es este quien procura el movimiento continuo de interpretación y comprensión. Esto permite ampliar las formas de producción de conocimiento, al otorgar especial atención a quienes loproducen a partir de su experiencia particular.

Por todo ello, decidimos utilizar la tradición hermenéutica, ya que permite cuestionar la aparente postura neutral y de poder del investigador. En ese sentido, en esta investigación el objeto de estudio es móvil, dinámico localizando en los sujetos y las organizaciones agentes que producen conocimiento. Como resultado, la diferencia entre investigador e investigado se disuelve para procurar procesos de comprensión amplios.

En consecuencia, el investigador deja de ser el sujeto que observa al otro (su objeto), para operar como un sujeto que al mismo tiempo que investiga, es investigado. Ese investigador- investigado debe anunciarse como tal, de modo que pueda articular sus intereses con elotro, quien ahora es su par epistémico en la investigación (Ruiz, 2017). Esa dualidad permite que, al mismo tiempo que se desarrolla la reflexión del estudio, se develen las prácticas, creencias, deseos, intereses y motivaciones del investigador-investigado (Harding, 1998).

Es así como se configuran múltiples agentes activos de la investigación, quienes no solo narran su experiencia, sino comprenden e interpretan su propia situación (Herrera, 2010). En ese sentido, sus relatos son textos cargados de poder (Haraway, 1995), para la construcción de conocimiento. El investigador y el investigado se asumen como pares epistémicos en la producción de textos. Textos que para el estudio resultan en la parte central de la producción de conocimiento.

Como consecuencia, y para lograr la interpretación hermenéutica, se apela a las siguientes herramientas para recabar la información:

- Revisión de documentos tales como la Política pública nacional para las familias colombianas 2012-2022 (2012) lineamientos de política pública para la familia de varios de los municipios donde se encuentran las universidades en mención, así como documentos de las instituciones de Educación superior.

- Entrevistas semiestructuradas a seis investigadores de cuatro programas de formación posgradual en familia, específicamente de la Universidad de Caldas en la ciudad de Manizales, la Universidad de Antioquia en Medellín, la Fundación Unimonserrate en Bogotá y la Universidad del Valle en Cali.

- Grupos focales -cuatro- con maestros de los programas posgraduales en familia de las IES mencionadas anteriormente.

- Entrevistas a profundidad con un hacedor o asesor de política pública en familia. 
Ruiz y Munévar-Vargas

Las entrevistas y grupos focales se transcribieron. Transcripciones que se incorporaron en Atlas-ti, versión 7.0. Dicho software facilita el análisis de datos cualitativos a partir de procesos como la codificación, escritura de memos, citas, construcción de redes y recuperación de datos; con esta herramienta se logra analizar y evaluar a profundidad la información. Es por ello que se logra un sinnúmero de relaciones junto a los hallazgos bibliográficos o soportes teóricos.

\section{Hallazgos}

A partir de lo anterior se logra un primer insumo de análisis que pretende compartir la comprensión en torno al vínculo entre el mundo de la Academia y la investigación en familia, junto al de las políticas públicas para las familias en Colombia, como se presenta a continuación:

Figura 1. Relación entre academia y política pública en familia

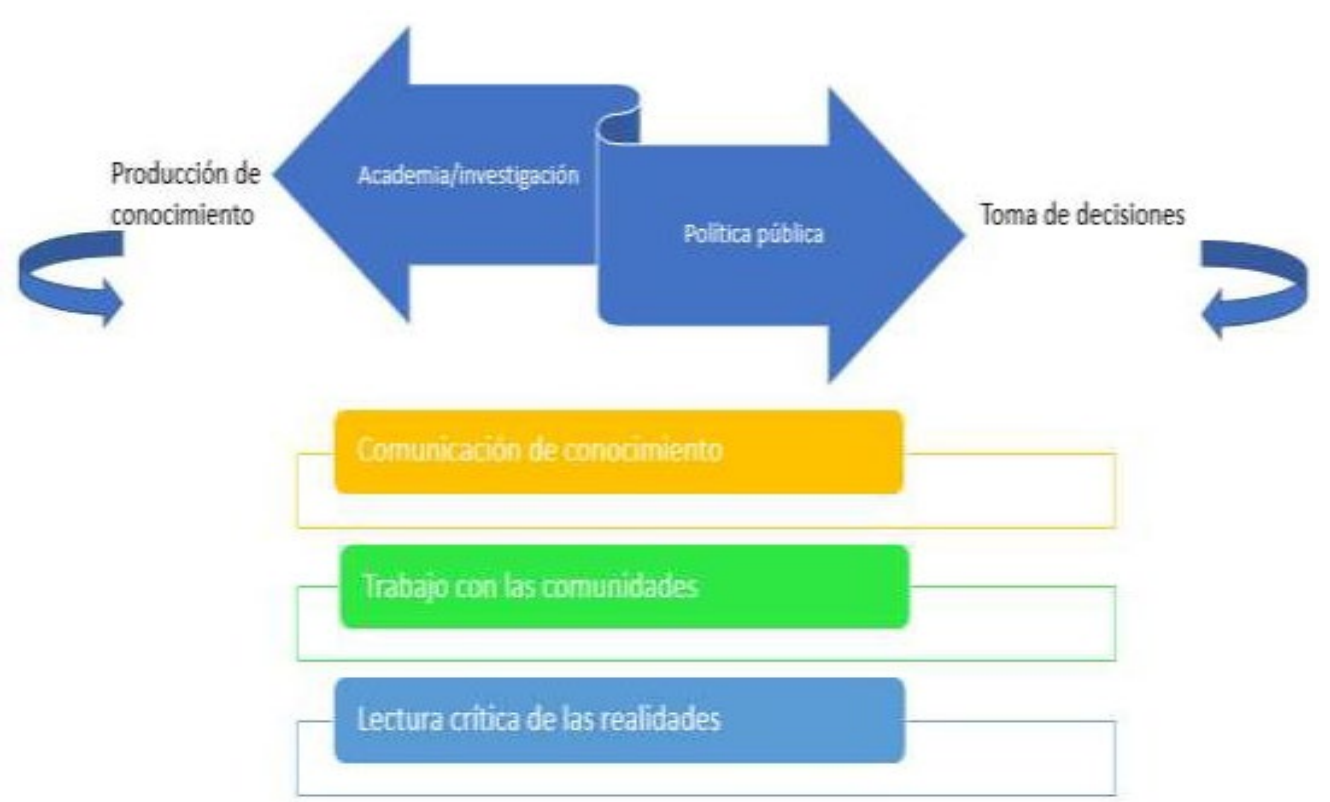

Fuente: elaboración propia.

\subsection{La Universidad produce, articula y resignifica el conocimiento}

El impacto logrado por la investigación, como producción de conocimiento y comunicación de este en torno a decisiones de política pública, en varios países de Europa y de América Latina es diferencial, tal es el caso del Reino Unido y Suecia, en donde es insumo importante y necesario, igualmente ocurre en los Estados Unidos (Saizarbitoria, 1987). Para América Latina, organismos como el Banco de Desarrollo en su informe 2015 presenta la necesidad de articular la investigación con las decisiones en política pública. 
Decisiones que, para el caso colombiano, y con especial atención en las ciencias sociales, establecen varios elementos para tener en cuenta. Específicamente en el caso de las políticas públicas de familia, en las cuales son las Instituciones de Educación Superior las que aportan y generan toda una serie de insumos, asumidos o no, por lo hacedores de políticas, que se consolidan como elementos funcionales, necesarios y vitales para la política pública de familias en Colombia. En el presente estudio se ratifica el papel de los maestros, de estudiantes de programas como los de Trabajo Social, y de estudiantes de programas posgraduales en familia; así como el de las comunidades impactadas por proyectos de extensión, y de investigación de los diversos programas que asumen las universidades asociadas en el estudio. De allí resaltamos los siguientes aspectos en torno a los actores y sus impactos logrados:

\section{Maestras y maestros, "tejedores" de conocimiento}

El papel desarrollado por los maestros y maestras que trabajan en las cuatro IES: Universidad de Caldas, Universidad del Valle, Universidad de Antioquía y Fundación Unimonserrate, guardan varios elementos en común que posibilitan la construcción social del conocimiento así como la gestión del mismo. Ello a partir de altos niveles de apropiación, no solo de su labor como integrantes de una comunidad académica, sino como seres humanos sensibles, frente a los temas de familia. El quehacer de la Universidad en Colombia, está cobijado por la ley 30, la cual entre otros anuncios plantea las funciones de la educación superior: investigación, docencia y extensión-proyección social, funciones todas que recaen en el ejercicio que desarrollan maestras y maestros universitarios, tanto en el caso de las IES públicas como privadas.

Para el caso de docentes, quienes integran los programas posgraduales del campo de familia, y en su mayoría alternan gran parte de su labor en los programas de pregrado de las mismas IES (especialmente en programas de Trabajo Social o de otros programas de las ciencias sociales y humanas), asumen en su quehacer académico las tres funciones: investigan, enseñan y asumen proyectos de proyección social. Lo anterior confluye en un nivel de comprensión ampliamente refinado del campo de estudio (Familia), con una extensa consideración de la historicidad de dicho campo y su conexión con los territorios; adicionalmente, son las maestras y maestros del campo de familia de las cuatro universidades del estudio, quienes son llamados y consultados para el desarrollo de políticas públicas locales e hicieron parte de la mesa consultiva para el desarrollo de la política pública nacional de las familias para Colombia. Sumado a ello, las maestras y maestros que hicieron parte del estudio, reconocen sus territorios y la complejidad de las familias. Es decir, son estas maestras y maestros en quienes radica una comprensión del campo de familia, pero se adiciona la sensibilidad que poseen en torno a los problemas sociales, al desarrollo de lo humano y las consecuencias de ello en las familias de Colombia. 
Ruiz y Munévar-Vargas

En síntesis las y los maestros que investigan en familia, en torno a temas como la diversidad familiar, el cuidado como consecuencia de la organización familiar, los roles de los sujetos en las familias, las ubicaciones socioculturales de las infancias y juventudes en la familia, la familia y la escuela como instituciones de socialización y cuestiones derivadas del campo de familia, como por ejemplo, el descentramiento de los roles de la mujer y el hombre, el papel del adulto mayor y las relaciones intergeneracionales, hace que productos de dichas investigaciones sean reconocidos en la lógica de producción de investigaciones, opciones de grado, semilleros de investigación, entre otros. Todo lo cual es llevado al territorio en esa conjunción entre las funciones de la educación superior, y que permite que maestras y maestros de dichos programas tengan contacto con instituciones de orden público que reconocen el papel de las y los investigadores, para ser consultados en el ejercicio del desarrollo de políticas públicas.

De forma que, la comprensión como espacio para la construcción social del conocimiento en familia, sumado a la sensibilidad en torno al campo de familia, configura un grupo especial de académicos e intelectuales en familia en Colombia, quienes no solo están autorizados para producir y comunicar el conocimiento en el campo, sino para coadyuvar en las decisiones que se deben asumir en el desarrollo de las políticas públicas de las familias en Colombia. Lo anterior, se evidencia con tres maestras y un maestro universitarios de la Universidad de Caldas, Universidad del Valle, Universidad de Antioquía y la Fundación Universitaria Unimonserrate. Son estas tres maestras y un maestro quienes han sido consultados para el desarrollo de políticas públicas de las ciudades de Medellín, Manizales, Bogotá y Cali y quienes a su vez hicieron parte desde sus IES, en la Mesa nacional de política pública para las Familias en Colombia.

El resultado, pareciera lógico, en torno a la integración de las instancias consultivas en el tema de política pública para las Familias, a maestras y maestro quienes por muchos años han desarrollado investigaciones y producciones en torno a las familias en el país. A ello se suma un múltiple ejercicio de conexión entre lo que implica la producción de conocimiento en un área o campo especifico y la afirmación de un sujeto, maestro o maestra, quien desarrolla a partir de su ejercicio de docencia e investigación, un conocimiento amplio de la realidad que circunda el campo. Campo queamplían en las investigaciones, y que como se ha mencionado responde a un despliegue de capacidades, de parte de las y los investigadores, así como a la sensibilidad, de su parte por problemáticas cotidianas que viven las familias en Colombia y se convierten en insumo para la producción de conocimiento y, por supuesto, para las posturas en las consultorías realizadas en los espacios de construcción de política pública.

Las administraciones nacionales y locales reconocen el valor de la Academia y el gran aporte que han realizado a lo largo de la historia colombiana en el estudio de familia, universidades como la Universidad de Caldas, la Universidad del Valle, la Universidad de 
Antioquia y la Fundación Unimonserrate, entre otras, han sido convocadas a la construcción de política pública y han alcanzado importantes contribuciones en el plano disciplinario, investigativo, social y político. Contribuciones que deben extenderse y encontrar retornos curriculares en los programas posgraduales de familia en las IES

Es así como, la Academia ha aportado un conocimiento disciplinar que ha puesto en diálogo a la política pública con el pensamiento mundial y nacional, a través de las diversas publicaciones que han generado una conversación constructiva con diversos autores y corrientes sociológicas, filosóficas y antropológicas haciendo de Colombia una nación fuerte en los discursos académicos con respecto al campo de familia. La Academia ha hecho de la familia en Colombia una cuestión a nivel internacional, con estudios de gran profundidad e impacto en las comunidades.

Otra contribución de la Academia ha sido el contacto directo con diferentes tipos de población, urbana y rural de Colombia, constituyéndose en un articulador entre quienes deciden la política pública y la realidad social, política, cultural y económica de las familias en Colombia. La Academia a través de sus docentes investigadores, de sus estudiantes de pregrado y posgrado se constituye en un aliado imprescindible para comprender el fenómeno de la familia en nuestra nación, tanto en el plano nacional como regional.

\section{Trabajo Social, emergencia de lo interdisciplinar}

El Estado colombiano, según varios de nuestros maestros y maestras acompañantes en la investigación, tiene una gran deuda de reconocimiento a la labor realizada al interior de los programas de Trabajo Social y especialmente, respecto al desarrollo y evolución de la temática de familia. Como se ha mencionado el impacto de la labor del maestro y maestra, se extiende por las regiones, desarrollando una tarea esencial para la configuración de las temáticas asociadas a las familias en términos disciplinares, contextuales y epistemológicos.

Es de reconocer el aporte de los programas de Trabajo Social a los asuntos de familia y que tienen en su principio constitutivo la transformación social como tarea; es así como la Academia desde el Trabajo Social desarrolla una labor de responsabilidad social de carácter ético con las comunidades. Como resultado, la investigación apunta a la transformación de diversos fenómenos sociales, los cuales devienen en modos de incidencia en la política pública en familia. Campo que resulta de una alta sensibilidad en nuestra nación y que requiere un trabajo dedicado y concienzudo.

No obstante, la producción académica en la universidad debería establecer un espacio importante para la investigación sobre la política pública. Esta tarea que puede parecer en primera instancia de baja influencia, se inserta de manera importante en razón a que la política pública necesita de una fase evaluativa, la cual puede ser articulada en los trabajos 
de los programas posgraduales, tales como los de maestría y de especialización, generando reflexiones que pueden servir como base fundamental para futuros proyectos de formulación de política pública social. El trabajo de las IES es un aporte importante en términos evaluativos, por ello es urgente una articulación con los hacedores de política pública quienes puedan revisar este aporte de tipo evaluativo y reflexivo y el cualconfigura, no solo un insumo sino un trabajo de madurez y seriedad importante, para las comunidades. No se puede olvidar que la política pública siempre deberá ser formulada con la conciencia clara de su contingencia y de una dinámica que debe acompañar los procesos sociales de la comunidad.

También se reconoce al programa de Trabajo Social en la tarea de inter disciplinarización de la cuestión familiar vinculando otras disciplinas como sociología, psicología, administración, entre otras, al discurso reflexivo y crítico de la cuestión familiar; también el programa de Trabajo Social ha introducido en varios programas la temática de familia; así como la creación y acompañamiento de diferentes programas de posgrado en torno a familia y la constitución de grupos y líneas de investigación y de intervención.

Las universidades del Valle, de Antioquia, Caldas y la Unimonserrate son reconocidas por el desarrollo y los aportes, desde Trabajo Social, al tema de tipologías familiares, arreglos familiares, formas familiares, familias de diferentes tipos y en los últimos años se viene trabajando el tema de la diversidad familiar, con la emergencia de rupturas de las tipologías tradicionales, provocando el reconocimiento de múltiples formas de asociaciones familiares, de quienes hacen parte sujetos que se desarrollan y están en el escenario público. Sumado a ello, está la cuestión de relaciones familiares y parentesco que se relaciona en los cursos en que desarrollan los contenidos basados en políticas públicas en familia.

\subsection{La toma de decisiones para la política pública en familia}

Es así como la Academia nos puede colocar en alerta frente a esta situación que está ocurriendo, lo cual se hizo evidente en algunos lugares que han hecho un ejercicio un poco más juicioso como lo hicimos nosotros, en la segunda administración de Antanas Mockus, donde se hizo el primer ejercicio de formulación participativa de política pública para familia. (A.M. Robledo, comunicación personal, 2017)

Una de las agentes de la investigación es una excandidata a la presidencia de la República quien también ha sido congresista y está en diálogo permanente con los centros de Investigación y Universidades. Ella reconoce varios insumos de origen en las Universidades, y en los centros de Investigación, para la construcción de la política pública en y para las familias. Además de establecer cómo en la dinámica que acompaña la política pública, la Academia tiene una misión crítica desde el contacto investigativo y la intervención que realiza en comunidades. Es claro que la política pública tiene una vigencia y que poner en cuestión, no es echar abajo todo lo construido, es repensar las cuestiones fundamentales, 
Ruiz y Munévar-Vargas

los principios metafísicos o la agenda política desde la cual se piensa la familia. Principios todos que gozan de fundamento social y político a partir de la investigación.

Para la representante, la democracia y la participación de los diferentes actores es necesaria para configurar una política pública que responda al reto social actual. Sin embargo, para ella el Estado debería entender su lugar en la generación de política pública, lugar que debe provocar la ubicación y participación de los diferentes actores en el espacio que corresponde. El diálogo de actores debe ser en términos democráticos y participativos, pero no puede ser el lugar de un campo de batalla paradigmático en el cual cada actor considere que está llamado a definir la política pública. Es precisamente en este punto donde la Academia tiene un lugar esencial como articulador del contexto social y de la revisión teórica, epistemológica, pero especialmente social y política, y que articule a la región, la nación y el mundo. La Academia y la investigación como agente vinculante de mundos, agentes y contextos diversos.

Finalmente, para la representante se hace importante el ejercicio de pedagogía, que ella asume, al interior del congreso y de las corporaciones públicas. Se parte, erradamente, al considerar que quienes ocupan cargos públicos tienen claridad sobre todos los temas que existen en la complejidad de la sociedad y no es así; es por ello que la estrategia de hacer pedagogía al interior del congreso, que podría pensarse ilógica, tiene un profundo impacto y se hace necesaria. Con ello la política incluye a todos los actores y particularmente a quienes tienen en sus manos el poder de decisión en la política pública, para el caso de familia.

De este modo, emergen varios puntos de encuentro entre el ejercicio investigativo y el de la consolidación de políticas públicas (ambos ejercicios cruzados por posturas ideológicas y por supuesto políticas). Uno de ellos se concreta en torno a la lectura crítica de las realidades que asumen nuestras comunidades en los territorios, lecturas que producen posturas. Otro elemento, es la consideración participativa de los diferentes agentes involucrados en los dos procesos, el de la investigación y el de las políticas públicas; y un tercer elemento transversal a todo ello, es la acción pedagógica como modo de "alfabetización" en torno a los procesos de académicos, como a los de construcción y apropiación de la política pública, para el caso del presente estudio en el campo de familia.

\section{Conclusiones}

Un aspecto para resaltar y que se ha trabajado poco, es el de la búsqueda de un estudio interdisciplinario sobre la familia. En la actualidad la epistemología no puede encerrarse en una sola forma de entender el conocimiento, menos en un fenómeno tan complejo como el de la familia. En este sentido se tiene una gran deuda con programas académicos como el de Trabajo Social, que ha llevado a la cuestión familiar diferentes disciplinas y aceptando el 
reto de estudiar la familia desde sus especificidades, han podido construir un discurso interdisciplinario en torno a la familia; en este sentido es muy rescatable esta labor, que se da por sentada, sin embargo, no todos los programas pueden afirmar el haber acompañado procesos de este tipo, máxime cuando algunos se hallan atrapados en la ilusión de la absoluta disciplinariedad.

La Academia tiene, entre muchos otros, dos retos fundamentales para continuar con su labor en la política pública para las familias: en primer lugar, formar actores sociales con amplia influencia en la implementación de la política pública, tarea que deberá contar con el apoyo del estado para alcanzar verdaderos resultados de madurez. En segundo lugar, y tal vez sea un reto aún mayor, es el de formar a la clase dirigente, esta tarea puede ser más compleja debido a la dificultad que tienen las clases dirigentes para dejarse acompañar y formar por el mundo académico, pues aunque sienten por él una gran admiración, consideran que el mundo académico es lejano a la realidad cotidiana; esta es una paradoja, debido a que esperan del mundo académico contacto con lo social, pero a la vez consideran que el mundo académico no entiende el mundo político, por ello urge establecer un diálogo franco y directo entre academia y ethos político.

En consecuencia, el principal reto de la Academia es insertarse, menos en las técnicas formales para la producción de conocimiento y más en el proceso de argumentación y consenso sobre las propuestas de política pública de familia. De acuerdo con Majone (1997) la tarea de los analistas consiste, en gran parte, en la producción de evidencias y argumentos para el debate público. Los analistas -como los abogados, los políticos y otras personas que hacen un uso funcional del lenguaje- deben estar familiarizados con todos los problemas técnicos del lenguaje, incluidos los retóricos.

Es por esta falencia discursiva y de convencimiento que evidenciamos problemas de implementación en políticas públicas ya cristalizadas institucionalmente. En donde es necesario entrar a pensar y discutir con esos otros decisores de políticas usualmente ignorados, como lo son las escalas inferiores de la burocracia, en donde existe amplia discrecionalidad y autonomía, a partir de las cuales se desarrollan juegos de influencias y negociaciones en torno a la aplicación de las normas y los procedimientos de la política de familia. De tal manera, sin su convencimiento, será inviable la implementación de cualquier política pública que sobre familia se haga en Colombia.

Es necesario que las administraciones nacionales y regionales comprendan su lugar en el desarrollo de la política pública para las familias, no se puede quedar en la contratación de expertos y elaboración de documentos, esto es una parte del trabajo, pero debe irse en mayor profundidad y asumir el compromiso de la implementación, evaluación y reformulación de la política pública. Por ello es indispensable que se tenga la valentía para romper paradigmas de tipo excluyente y trabajar por políticas más inclusivas. 


\section{Agradecimientos}

Con especial afecto y gratitud a las maestras María Cristina Palacio y Carolina Chalarca de la Universidad de Caldas, a Amparo Micolta de la Universidad del Valle, a Hernando Muñoz de la Universidad de Antioquía, a Ginna Luque de la Fundación Universitaria Unimonserrate, a Patricia Robledo y Ángela María Robledo por su humildad y gentileza en torno a este estudio. A todas y todos los que posibilitaron el tránsito de una inquietud investigativa a este insumo que posibilita algunas inquietudes y provocaciones a los y las estudiosas de la epistemología, del campo de familia y de los estudios de las políticas públicas. A todas y todos los que participaron en este estudio.

Dedicado: A las familias de Colombia y a los coequiperos de mi familia: Laura Weistein †, Nano, Luis Ángel † y Jonathan, un abrazo de total agradecimiento. Y por supuesto, a los que hacen academia en medio de las dificultades.

\section{Referencias bibliográficas}

Corte Constitucional de Colombia [C.C.]. (18 de febrero de 2015). Sentencia T-070/15. [MS Martha Victoria Sáchica Méndez].

Corte constitucional de Colombia [C.C.]. (2 de junio de 2016). Sentencia T-292/16. [MP Gabriel Eduardo Mendoza Martelo].

Delatin, D., Texeira, M., y Sandroni, L. (2015). De la investigación a la política pública: producción y circulación de conocimiento científico. Nómadas, (42), 151-165.

Espinosa, L., Rivera, L., y Ruiz, L. (2016). Experiencias de tres jóvenes hijos de familias homoparentales (Tesis de maestría). Fundación Universitaria Monserrate. Bogotá, Colombia.

Gadamer, H. G. (1993). Verdad y método. Fundamentos de una hermenéutica filosófica. Salamanca: Ediciones Sígueme.

Guevara, C., y Ortiz, M. I. (17 de septiembre de 2016). Más de 4300 niños, en medio del debate sobre la adopción. El tiempo. Recuperado de https://www.eltiempo.com/justicia/cortes/debate-sobre-adopcion-por-parejasgay-42574.

Haraway, D. (1991). Ciencias, ciborgs y mujeres. La reinvención de la naturaleza. Madrid: Ediciones cátedra.

Harding, S. (1998). ¿Existe un método feminista? En E. Bartra (Comp.), Debates en torno a una metodología feminista (pp. 9-34). México: Universidad Autónoma metropolitana.

Herrera, J. (2010). La comprensión de lo social. Horizonte hermenéutico de las ciencias sociales. Bogotá: Antropos.

Jiménez, J. (18 de enero de 2015). Se calienta el debate sobre adopción por parte de homosexuales. El espectador, Judicial. Recuperado de 
Ruiz y Munévar-Vargas https:/ / www.elespectador.com/judicial/se-calienta-el-debate-sobre-adopcion-porparte-de-homosexuales-article-538606/.

Laswell, H. (1971). Pre-View of Policy Sciences (Policy sciences book series). New York: American Elsevier.

Lozano, A. (2008). Aspectos sobre política pública: ejercicios e instrumentos para su análisis. Bogotá: Universidad Nacional de Colombia.

Majone, G. (1997) Evidencia, argumentación y persuasión en la formulación de políticas. México: Fondo de Cultura Económica.

Ministerio de Salud y Protección Social. (2012). Política pública nacional para las familias colombianas 2012-2022. Bogotá, Colombia: Ministerio de Salud y Protección Social.

Roth-Deubel, A. (2002). Políticas públicas. Formulación, implementación y evaluación. Bogotá: Universidad Nacional de Colombia.

Ruiz, J. (2017). Ser y estar transgénero: jóvenes transgénero y acción colectiva (Tesis doctoral). Universidad de Manizales. Manizales, Colombia.

Saizarbitoria, R. (1987). Investigación social y políticas públicas. Revista de servicios sociales, (2), 1134-1145.

Sousa-Santos, B. de. (2003). Crítica de la razón indolente: Contra el desperdicio de la experiencia: Para un nuevo sentido común. Bilbao: Editorial Desclée Brouwer.

Subirats, J., Knoepfel, P., Larrue, C., y Varone, F. (2008). Análisis y gestión de políticas públicas (pp. 31-69). Barcelona: Ariel.

258 Torres-Melo, J., y Santander, J (2013). Introducción a las políticas públicas: conceptos y herramientas desde la relación entre Estado y ciudadanía. Bogotá: IEMP.

Touraine, A. (2006) ¿Podremos vivir juntos? México: Fondo de cultura económica. 


\section{OTROS ARTÍCULOS DE PROSPECTIVA No. 32 DE 2021}

\section{EDITORIAL}

Compromisos, dilemas y desafíos del Trabajo Social con dimensión colectiva en tiempos de pandemia

Enrique Pastor-Seller

\section{ARTÍCULOS}

La narrativa biográfica como alternativa para la comprensión del Conflicto y la violencia en Colombia: una experiencia pedagógica universitaria

Sara Sofía Castaño-Barco y Claudia BermúdezPeña

Experiencias de transición a la vida adulta de jóvenes que migraron solos. Un estudio en Aragón (España) Daniel Jiménez-Franco; Raquel Berzosa-Callén y Chabier Gimeno-Monterde

Fundamentals of Education in Intervention with Young Offenders. A Critical Review of a 'Successful' Case in Andalucía, España

David Herrera-Pastor

Subjetividad juvenil: lecturas desde y para el Trabajo Social en Colombia

Daniela Joya-Valbuena

Participación politica de los jóvenes del Valle del Cauca, Colombia, en la toma de decisiones públicas

Luis Eduardo Gutiérrez-Rojas y Amanda Ramírez-Giraldo

Victimización y perpetración de violencia en pareja adolescente y redes de apoyo en Colombia. Análisis con perspectiva de género

Johanna Alexandra Reina-Barreto
Apoyo social percibido, autoestima y maternidad adolescente: entre el respeto y la intrusión. Estudio en Traiguén, Chile

Gloria Mora-Guerrero, Luisa Escárate-Colín, Carol Espinoza-Lerdón y Andrea Peña-Paredes

El desarrollo en el Trabajo Social de Colombia: un campo transversal, disperso y polivalente

Maira Judith Contreras-Santos

Análisis de la situación laboral de las alimentadoras en la caficultura de la zona central colombiana, a la luz de la teoría de Nancy Fraser

Pablo Andrés Arango-Giraldo

Prioridades de investigación en la Obra social de empleados públicos, Mendoza, Argentina. Un estudio Delphi sobre producción de conocimientos en la seguridad social

Cecilia Amalia Molina

Producción de conocimiento y toma de decisiones. Relaciones entre academia y política pública para las familias en Colombia

José Raúl-Ruíz y Sandro Leonardo MunévarVargas

El staff de prácticas profesionales en Trabajo Social: espacio de fomento del aprendizaje autodirigido. Un estudio de caso en la Universidad Católica Luis Amigó, Colombia

Cristian Eduardo Blanco-García

\section{ARTISTA INVITADO}

Margie Reinel-Aguilar Ao Cicadidae

Prospectiva

\section{PROSPECTIVA Revista de Trabajo Social e Intervención Social}

No. 32 • jul.-dic. 2021

e-ISSN: 2389-993X • Universidad del Valle 